\title{
Efficient Procedures of Sensitivity Analysis for Structural Vibration Systems with Repeated Frequencies
}

\author{
Shijia Zhao, Tao Xu, Guikai Guo, Wei Zhang, and Dongkai Liu \\ College of Mechanical Science and Engineering, Jilin University, Changchun 130022, China \\ Correspondence should be addressed to Guikai Guo; ggk1218@gmail.com
}

Received 19 May 2012; Revised 20 November 2012; Accepted 18 December 2012

Academic Editor: Marco H. Terra

Copyright (c) 2013 Shijia Zhao et al. This is an open access article distributed under the Creative Commons Attribution License, which permits unrestricted use, distribution, and reproduction in any medium, provided the original work is properly cited.

\begin{abstract}
Derivatives of eigenvectors with respect to structural parameters play an important role in structural design, identification, and optimization. Particularly, calculation of eigenvector sensitivity is considered when the eigenvalues are repeated. A relaxation factor embedded in the combined approximations (CA) method makes it effective to the structural response at various modified designs. The proposed method is feasible after overcoming the defection of irreversibility of the characteristic matrix. Numerical examples show that it is easy to implement the computational procedure, and the method presented in this paper is efficient for the general linear vibration damped systems with repeated frequencies.
\end{abstract}

\section{Introduction}

Many engineering optimization problems, for example, model updating [1] or structural damage detections [2], lead to a sensitivity analysis of eigenproblems. As a result, the study of the sensitivity of eigensolutions due to variations in the system parameters has been an important research area. A dynamic model can be far from the assumed prototype because there is usually a variation, such as a mistuned parameter or a geometrical irregularity. For these reasons, sensitivity analysis is meaningful to perform a theoretical study and give a guide for engineering practice. Two main difficulties exist in computing the eigenvectors derivatives. One of the main difficulties is how to change the irreversible state of characteristic matrix. The other difficulty is how to establish a uniform efficient method for computing the eigenvectors derivatives with repeated eigenvalues.

In the area of eigenproblem sensitivity, there is a great deal of interest and significant progress in sensitivity of problems with repeated eigenvalues. The situation of repeated frequencies occurs in complex large structures, such as an airplane, rocket, high tower, bridge and ocean platform. Wilkinson first put forward the mode expansion method which was of importance in the engineering [3]. Fox and Kapoor [4] provided the expressions for derivatives of eigensolutions with respect to any design variable by the mode expansion method. The expressions are valid for symmetric undamped systems and have been used in a wide range of application areas of structural dynamics. Rogers [5] has extended Fox and Kapoor's method to calculate the first-order derivative of eigenvectors for more general asymmetric. Nelson [6] proposed a very efficient algorithm for eigenvector derivatives that only requires those eigensolutions information which is to be differentiated. However, this method cannot deal with cases of repeated eigenvalues which can often occur in many practical engineering problems. Many researches [7-10] have applied Nelson's approach to the symmetric eigensystems with repeated eigenvalues for computing the derivatives of eigensolutions; moreover, [10] pointed that a critical step in Dailey's method may fail under certain circumstances. Lee et al. [11] derived an iterative method for sensitivity analysis of eigenvectors with distinct and repeated eigenvalues in the generalized eigenproblems. Chen [12] developed matrix perturbation theory to determine the first part of the repeatedroot eigenvector derivatives. The implementation effort must be weighed against the performance of the algorithms as reflected in their accuracy and computational efficiency.

In choosing a suitable sensitivity analysis method for the system with repeated eigenvalues, the following two factors should be well balanced: the accuracy of the calculations and 
the computational effort involved. High accuracy, however, is often achieved at the expense of more computational effort. The CA approach is the most suitable for efficientaccurate evaluation of the structural response at various modified designs $[13,14]$. A relaxation factor embedded in the CA method is to keep the reversibility of the characteristic matrix which makes it possible and effective to deal with the problems of sensitivity analysis for multiple eigenvalues. To preserve the ease of implementation and the advantage of the relaxation factor, improving significantly the quality of the results, extension of CA method to sensitivity analysis for the systems with repeated eigenvalues is presented in this paper.

The purpose of Section 2 is to give a brief background of modal sensitivity analysis. An overview of the CA approach is given in Section 3. An extended CA method for solving the first-order derivatives with repeated eigenvalues is developed systematically in Section 4. Numerical examples are demonstrated in Section 5, and the conclusions are drawn in Section 6 .

\section{Theoretical Background}

The eigenproblem of a linear vibration damped system can be expressed as

$$
\left(\lambda^{2} \mathbf{M}+\lambda \mathbf{C}+\mathbf{K}\right) \mathbf{u}=\mathbf{0},
$$

where $\mathbf{M}, \mathbf{C}$, and $\mathbf{K} \in \mathbb{C}^{n \times n}$ are the mass, damping, and stiffness matrices, respectively, and $(\lambda, \mathbf{u})$ is the eigenpair of the system. Denote $2 n=N$ briefly.

$$
\text { Let } \mathbf{z}=\left(\begin{array}{c}
\mathbf{u} \\
\lambda \mathbf{u}
\end{array}\right)_{N \times 1} \text { and } \mathbf{A}=\left(\begin{array}{cc}
\mathbf{0} & \mathbf{I}_{n} \\
-\mathbf{M}^{-1} \mathbf{K} & -\mathbf{M}^{-1} \mathbf{C}
\end{array}\right)_{N \times N} \text {, and } \mathbf{z} \text { and } \mathbf{A}
$$
are the state vector and the state matrix, respectively. We can verify

$$
\mathbf{A z}=\lambda \mathbf{z}, \quad i=1,2, \ldots, N,
$$

where $\lambda=\omega^{2}, \omega$ is the natural frequency.

It can be called a system with repeated frequencies if eigenproblem has repeated eigenvalues. Therefore, researches on the close frequencies are equal to those on close eigenvalues. In the following, we give the definitions for classifying the nondefective system and the defective system. The system will be defective if the algebra multiplicity of the eigenvalue $\lambda$ is greater than the geometric multiplicity; therefore the defective system has an incomplete set of eigenvectors to span the state space. The system must be nondefective if $\lambda$ is distinct eigenvalue or the algebra multiplicity of the eigenvalue $\lambda$ is equal to geometric multiplicity. The derivatives of nondefective systems with repeated frequencies are presented in this study.

\section{CA Method}

The reanalysis problem to be solved for each modified design can be stated briefly as follows.

Given an initial symmetric positive-definite stiffness matrix $\mathbf{K}_{0}$ and the load vector $\mathbf{R}_{0}$, the initial displacements $\mathbf{r}_{0}$ are calculated by

$$
\mathbf{K}_{0} \mathbf{r}_{0}=\mathbf{R}_{0} .
$$

The initial stiffness matrix $\mathbf{K}_{0}$ is usually given in the decomposed form

$$
\mathbf{K}_{0}=\mathbf{U}_{0}^{T} \mathbf{U}_{0}
$$

where $\mathbf{U}_{0}$ is the upper triangular matrix.

Assume a change in the structure and the corresponding changes $\Delta \mathbf{K}$ in the stiffness matrix and $\Delta \mathbf{R}$ in the load vector, where $\Delta \mathbf{K}$ and $\Delta \mathbf{R}$ might be due to both design (or optimization) considerations and analysis (or nonlinearity) considerations. The object is to estimate the modified displacements $\mathbf{r}$ due to the changes in the structure without solving the complete set of modified analysis equations:

$$
\mathbf{K r}=\mathbf{R},
$$

where $\mathbf{K}=\mathbf{K}_{0}+\Delta \mathbf{K}, \mathbf{R}=\mathbf{R}_{0}+\Delta \mathbf{R}$.

(i) The matrix of basis vectors $\mathbf{r}_{B}$ is determined by the binomial series

$$
\begin{gathered}
\mathbf{r}_{1}=\mathbf{K}_{0}^{-1} \mathbf{R}, \\
\mathbf{r}_{i}=-\mathbf{K}_{0}^{-1} \Delta \mathbf{K r}_{i-1}, \quad i=1,2, \ldots, s, \\
\mathbf{r}_{B}=\left(\mathbf{r}_{1}, \mathbf{r}_{2}, \ldots, \mathbf{r}_{s}\right),
\end{gathered}
$$

where the preselected $s$ is assumed to be much smaller than the number of degree-of-freedom (DOF) $n$.

(ii) Through the matrix of basis vector $\mathbf{r}_{B}$, we compute condensed stiffness matrix $\mathbf{K}_{R}$ and mass matrix $\mathbf{R}_{R}$, where $\mathbf{K}_{R}$ and $\mathbf{R}_{R}$ are defined as

$$
\mathbf{K}_{R}=\mathbf{r}_{B}^{T} \mathbf{K} \mathbf{r}_{B}, \quad \mathbf{R}_{R}=\mathbf{r}_{B}^{T} \mathbf{R} .
$$

(iii) The coefficient vector $\mathbf{y}$ can be calculated by solving a reduced set of $s$ th order reanalysis equations instead of computing the exact solution by solving the larger $n$th order system:

$$
\mathbf{K}_{R} \mathbf{y}=\mathbf{R}_{R}
$$

where $\mathbf{y}^{T}=\left\{y_{1}, y_{2} \ldots, y_{s}\right\}$.

(iv) The approximate displacements vector $\mathbf{r}$ is evaluated by a linear combination of matrix of basis vector $\mathbf{r}_{B}$ and the coefficient vector $\mathbf{y}$ :

$$
\mathbf{r}=y_{1} \mathbf{r}_{1}+y_{2} \mathbf{r}_{2}+\cdots+y_{s} \mathbf{r}_{s}=\mathbf{r}_{B} \mathbf{y} .
$$

In large-scale structural design and optimization problems, the cost of reanalysis, even for a small change in the design, is significant. The CA approach is efficient in the reanalysis problems of large structures, and high quality approximations can be achieved with a small effect for changes in design variables. 


\section{Sensitivity Analysis for Repeated Frequencies}

The eigenproblem corresponding to the state matrix $\mathbf{A}$ is

$$
\mathrm{A} \Theta=\Theta \mathrm{J}
$$

where $\mathbf{J}$ is the Jordan canonical form of matrix $\mathbf{A}$,

$$
\begin{gathered}
\mathbf{J}=\left(\begin{array}{cccc}
\mathbf{J}_{1} & & & \\
& \mathbf{J}_{2} & & \\
& & \ddots & \\
& & & \mathbf{J}_{t}
\end{array}\right), \quad(1 \leq t<N), \\
\mathbf{J}_{i}=\left(\begin{array}{cccc}
\lambda_{i} & 1 & & \\
& \lambda_{i} & & \\
& & \ddots & 1 \\
& & \lambda_{i}
\end{array}\right)_{m_{i} \times m_{i}}, \quad\left(\sum_{i=1}^{t} m_{i}=N\right),
\end{gathered}
$$

$m_{i}$ is the multiplicities of eigenvalues $\lambda_{i}$. $\Theta$ is called the eigenmatrix of the state matrix $\mathbf{A}$.

Suppose that multiplicity of the eigenvalue $\lambda$ is $t(2 \leq$ $t \leq N)$; the remaining eigenvalues are distinct, that is, $\lambda, \ldots, \lambda, \lambda_{t+1}, \lambda_{t+2}, \ldots, \lambda_{N}$. Equation (11) becomes

$$
\mathbf{J}=\left(\begin{array}{cc}
\mathbf{J}_{t} & \mathbf{0} \\
\mathbf{0} & \mathbf{J}_{N-t}
\end{array}\right)
$$

where

$$
\begin{aligned}
& \mathbf{J}_{t}=\left(\begin{array}{cccc}
\lambda & 1 & & 0 \\
& \lambda & & \\
& & \ddots & 1 \\
0 & & & \lambda
\end{array}\right)_{t \times t}, \\
& \mathbf{J}_{N-t}=\left(\begin{array}{ccc}
\lambda_{t+1} & & 0 \\
& \lambda_{t+2} & \\
0 & & \lambda_{N}
\end{array}\right)_{(N-t) \times(N-t)} .
\end{aligned}
$$

If the structural parameters have small changes, such that the state matrix $\mathbf{A}$ has a change $\Delta \mathbf{A}$, for the modified state matrix $\mathbf{A}+\Delta \mathbf{A}$, there exists an invertible matrix $\tilde{\boldsymbol{\Theta}}$, such that

$$
(\mathbf{A}+\Delta \mathbf{A}) \tilde{\boldsymbol{\Theta}}=\tilde{\boldsymbol{\Theta}} \tilde{\mathbf{J}}
$$

where $\tilde{\mathbf{A}}=\mathbf{A}+\Delta \mathbf{A}, \tilde{\mathbf{J}}=\mathbf{J}+\Delta \mathbf{J}, \tilde{\mathbf{J}}$ is the new Jordan canonical form of the state matrix $\tilde{\mathbf{A}}$.

Note

$$
\mathbf{L}_{0}=\mathbf{A}-\lambda \mathbf{I}+\rho \mathbf{I}, \quad \Delta \mathbf{L}=\Delta \mathbf{A}-\Delta \mathbf{J}-\rho \mathbf{I}, \quad \mathbf{L}=\mathbf{L}_{0}+\Delta \mathbf{L} .
$$

Combine (16) and (17), it can be derived that

$$
\left(\mathbf{L}_{0}+\Delta \mathbf{L}\right) \tilde{\boldsymbol{\Theta}}=\tilde{\boldsymbol{\Theta}} \mathbf{B},
$$

where $\mathbf{B}=\left(\mathbf{0}, \mathbf{e}_{1}, \ldots, \mathbf{e}_{N-1}\right), \mathbf{e}_{i}(1 \leq i \leq N-1)$ is the unit vector.
Expand (18) and rewrite characteristic equations to the form of equivalent equations:

$$
\begin{gathered}
\left(\mathbf{L}_{0}+\Delta \mathbf{L}\right) \tilde{\psi}_{1}=\mathbf{0}, \\
\left(\mathbf{L}_{0}+\Delta \mathbf{L}\right) \tilde{\psi}_{i}=\tilde{\psi}_{i-1}, \quad i=2,3, \ldots, N .
\end{gathered}
$$

The selection of relaxation factor should guarantee that $\mathbf{L}_{0}(=\mathbf{A}-\lambda \mathbf{I}+\rho \mathbf{I})$ is invertible. It is an equivalent technology, and the value of $\rho(\rho \neq 0)$ does not affect the results.

For $i=1$ in (19), we can get the generalized eigenvector $\tilde{\boldsymbol{\theta}}_{1}$.

Based on the CA approach, we obtain

$$
\tilde{\boldsymbol{\theta}}_{i}^{k}=-\mathbf{L}_{0}^{-1} \Delta \mathbf{L} \tilde{\boldsymbol{\theta}}_{i}^{k-1}, \quad k=1,2, \ldots, s, i=2,3, \ldots, N .
$$

From (20), the basis vectors can be given by

$$
\tilde{\boldsymbol{\theta}}_{i}^{B}=\left(\tilde{\boldsymbol{\theta}}_{i}^{1}, \tilde{\boldsymbol{\theta}}_{i}^{2}, \ldots, \tilde{\boldsymbol{\theta}}_{i}^{s}\right), \quad i=2,3, \ldots, N,
$$

where $s$ is the number of basis vectors. $\tilde{\boldsymbol{\theta}}_{i}^{B}$ and the coefficient vectors $\mathbf{y}_{i}$; it is derived that

The vector $\tilde{\boldsymbol{\theta}}_{i}$ is a linear combination of the basis vectors$$
\tilde{\boldsymbol{\theta}}_{i}=y_{i}^{1} \tilde{\boldsymbol{\theta}}_{i}^{1}+\cdots+y_{i}^{\tilde{\boldsymbol{\theta}}_{i}^{s}}, \quad i=2,3, \ldots, N
$$

where the vectors of coefficient are determined

$$
\mathbf{y}_{i}=\left(y_{i}^{1}, y_{i}^{2}, \ldots, y_{i}^{s}\right)^{T}, \quad i=2,3, \ldots, N
$$

Let

$$
\mathbf{L}_{i}^{R}=\left(\tilde{\boldsymbol{\theta}}_{i}^{B}\right)^{T} \tilde{\boldsymbol{\theta}}_{i}^{B}, \quad \mathbf{R}_{i}^{R}=\left(\tilde{\boldsymbol{\theta}}_{i}^{B}\right)^{T} \tilde{\boldsymbol{\theta}}_{i-1}, \quad s, i=2,3, \ldots, N .
$$

Therefore, only to solve the smaller $s \times s$ system

$$
\mathbf{L}_{i}^{R} \mathbf{y}_{i}=\mathbf{R}_{i}^{R}, \quad i=2,3, \ldots, N
$$

we can get the vectors of coefficients $\mathbf{y}_{i}$, and the computation is much smaller than the original equations (18). Substituting the vectors of coefficient to (22) and repeating the above iterations for $i=2,3, \ldots, N$, the eigenvectors $\tilde{\boldsymbol{\theta}}_{i}$ can be computed. Summing up the previous ideas, the modified eigenvector matrix $\tilde{\Theta}$ can be obtained.

The approximate generalized eigenvector derivatives can be calculated by differentiating the approximate generalized eigenvectors expression (22) with respect to a design of variable $d$ :

$$
\frac{\partial \tilde{\boldsymbol{\theta}}_{i}}{\partial d}=\frac{\partial \tilde{\boldsymbol{\theta}}_{i}^{B}}{\partial d} \mathbf{y}_{i}+\tilde{\boldsymbol{\theta}}_{i}^{B} \frac{\partial \mathbf{y}_{i}}{\partial d}, \quad i=1,2, \ldots, N
$$


(1) Introduce the relaxation factor and transform the eigenequations into equivalent equations.

(2) Compute eigenvector $\widetilde{\psi}_{1}$.

(3) The eigenvectors $\widetilde{\boldsymbol{\theta}}_{i}(i=2,3, \ldots, N)$ can be expressed by the basis vectors $\widetilde{\boldsymbol{\theta}}_{i}^{B}(i=2,3, \ldots, N)$ and coefficients vectors $\mathbf{y}_{i}(i=2,3, \ldots, N)$.

(4) Compute $\partial \widetilde{\boldsymbol{\theta}}_{i} / \partial d=\left(\partial \widetilde{\boldsymbol{\theta}}_{i}^{B} / \partial d\right) \mathbf{y}_{i}+\widetilde{\boldsymbol{\theta}}_{i}^{B}\left(\partial \mathbf{y}_{i} / \partial d\right), i=1,2, \ldots, N$.

Algorithm 1: The produce of the proposed method.

Differentiating and rearranging (25), we obtain for $\partial \mathbf{y}_{i} / \partial d$,

$$
\mathbf{L}_{i}^{R} \frac{\partial \mathbf{y}_{i}}{\partial d}=\frac{\partial \mathbf{R}_{i}^{R}}{\partial d}-\frac{\partial \mathbf{L}_{i}^{R}}{\partial d} \mathbf{y}_{i}, \quad i=1,2, \ldots, N
$$

The matrix $\partial \mathbf{K}_{i}^{R} / \partial d$ and the vector $\partial \mathbf{R}_{i}^{R} / \partial d$ are evaluated by differentiating (24):

$$
\begin{gathered}
\frac{\partial \mathbf{L}_{i}^{R}}{\partial d}=\frac{\partial\left(\tilde{\boldsymbol{\theta}}_{i}^{B}\right)^{T}}{\partial d} \tilde{\mathbf{L}}_{i}^{B}+\left(\tilde{\boldsymbol{\theta}}_{i}^{B}\right)^{T} \frac{\partial \mathbf{L}}{\partial d} \tilde{\boldsymbol{\theta}}_{i}^{B}+\left(\tilde{\boldsymbol{\theta}}_{i}^{B}\right)^{T} \mathbf{L} \frac{\partial \tilde{\boldsymbol{\theta}}_{i}^{B}}{\partial d} \\
\frac{\partial \mathbf{R}_{i}^{R}}{\partial d}=\frac{\partial\left(\tilde{\boldsymbol{\theta}}_{i}^{B}\right)^{T}}{\partial d} \tilde{\boldsymbol{\theta}}_{i-1}, \quad i=1,2, \ldots, N
\end{gathered}
$$

The algorithm for the first-order sensitivity analysis of eigenvectors for multiple eigenvalues is summarized in Algorithm 1.

The approximation quality and computation efficiency are usually two conflicting factors in selecting an approximate reanalysis method. This also holds in the approximation method presented. The number of algebraic operations (multiplication and division) needed to solve an $n \times n$ set of equations is $n^{3} / 3$. The operations cost for the CA method is $3 n^{2} s+n s^{2}+s^{3} / 3$, where $s$ is the number of basis vectors.

\section{Numerical Examples}

As an illustrative example in case of the structural vibration system with multiple eigenvalues, the 5 degrees of freedom (DOF) spring-mass mechanical system shown in Figure 1 are considered. It is assumed that only vibrations in the vertical plane are possible.

The mass parameters of the mass matrix $\mathbf{M}$ are

$$
\begin{aligned}
& m_{11}=m_{1}, \quad m_{22}=m_{2}, \quad m_{33}=m_{3}, \\
& m_{44}=J_{4}=\frac{m_{3} L^{2}}{12}, \quad m_{55}=J_{5}=\frac{m_{3} L^{2}}{12},
\end{aligned}
$$

where $J_{i}(i=4,5)$ is the moment of inertia, $L$ is the edge length of the rotation plan, and $\theta_{i}(i=4,5)$.
The springs have the following stiffnesses:

$$
\begin{gathered}
k_{11}=k_{1}, \quad k_{12}=-k_{1}, \quad k_{13}=k_{14}=k_{15}=0, \\
k_{22}=k_{1}+k_{2}, \quad k_{23}=-k_{2}, \quad k_{24}=k_{25}=0, \\
k_{33}=k_{2}+k_{3}+k_{4}+k_{5}+k_{6}, \\
k_{34}=\frac{L}{2}\left(k_{3}-k_{4}+k_{5}-k_{6}\right), \\
k_{35}=\frac{L}{2}\left(k_{3}+k_{4}-k_{5}-k_{6}\right), \\
k_{44}=\left(\frac{L}{2}\right)^{2}\left(k_{3}+k_{4}+k_{5}+k_{6}\right), \\
k_{45}=-\left(\frac{L}{2}\right)^{2}\left(k_{3}-k_{4}-k_{5}+k_{6}\right), \\
k_{55}=\left(\frac{L}{2}\right)^{2}\left(k_{3}+k_{4}+k_{5}+k_{6}\right) .
\end{gathered}
$$
follows:

The constituents of the damped matrix $\mathbf{C}$ are given as

$$
\begin{gathered}
c_{11}=c_{1}, \quad c_{12}=-c_{1}, \\
c_{13}=c_{14}=c_{15}=0, \quad c_{22}=c_{1}+c_{2}, \\
c_{23}=-c_{2}, \quad c_{24}=c_{25}=0, \\
c_{33}=c_{2}+c_{3}+c_{4}+c_{5}+c_{6}, \\
c_{34}=\frac{L}{2}\left(c_{3}-c_{4}+c_{5}-c_{6}\right), \\
k_{35}=\frac{L}{2}\left(c_{3}+c_{4}-c_{5}-c_{6}\right), \\
c_{44}=\left(\frac{L}{2}\right)^{2}\left(c_{3}+c_{4}+c_{5}+c_{6}\right), \\
c_{45}=-\left(\frac{L}{2}\right)^{2}\left(c_{3}-c_{4}-c_{5}+c_{6}\right), \\
c_{55}=\left(\frac{L}{2}\right)^{2}\left(c_{3}+c_{4}+c_{5}+c_{6}\right) .
\end{gathered}
$$


TABLE 1: Result of eigenvectors and the sensitivity analysis.

\begin{tabular}{|c|c|c|c|c|c|}
\hline & \multicolumn{5}{|c|}{ Mode } \\
\hline & 1 & 2 & 3 & 4 & 5 \\
\hline \multirow{10}{*}{$\begin{array}{l}\text { Generalized } \\
\text { eigenvectors }\end{array}$} & $\begin{array}{l}-1.6940 e-4 \\
-7.5774 e-2 i\end{array}$ & $\begin{array}{l}-1.6940 e-4 \\
+7.5774 e-2 i\end{array}$ & $\begin{array}{l}-5.9292 e-4 \\
-1.4832 e-1 i\end{array}$ & $\begin{array}{l}-5.9292 e-4 \\
+1.4832 e-1 i\end{array}$ & $\begin{array}{l}-8.8391 e-3 \\
-3.7710 e-1 i\end{array}$ \\
\hline & $\begin{array}{c}6.4403 e-5 \\
+6.6628 e-2 i\end{array}$ & $\begin{array}{c}6.4403 e-5 \\
-6.6628 e-2 i\end{array}$ & $\begin{array}{l}-7.6481 e-4 \\
-3.8049 e-2 i\end{array}$ & $\begin{array}{l}-7.6481 e-4 \\
+3.8049 e-2 i\end{array}$ & $\begin{array}{l}-9.1033 e-3 \\
-3.6559 e-1 i\end{array}$ \\
\hline & $\begin{array}{c}2.0564 e-4 \\
-1.8688 e-2 i\end{array}$ & $\begin{array}{c}2.0564 e-4 \\
+1.8688 e-2 i\end{array}$ & $\begin{array}{l}-3.9167 e-4 \\
+5.2447 e-2 i\end{array}$ & $\begin{array}{l}-3.9167 e-4 \\
-5.2447 e-2 i\end{array}$ & $\begin{array}{l}-9.5377 e-3 \\
-3.4587 e-1 i\end{array}$ \\
\hline & $\begin{array}{c}3.3666 e-5 \\
+1.4966 e-6 i\end{array}$ & $\begin{array}{c}3.3666 e-5 \\
-1.4966 e-6 i\end{array}$ & $\begin{array}{l}-1.7466 e-4 \\
-1.1910 e-5 i\end{array}$ & $\begin{array}{l}-1.7466 e-4 \\
+1.1910 e-5 i\end{array}$ & $\begin{array}{l}-1.0552 e-3 \\
+5.7213 e-5 i\end{array}$ \\
\hline & $\begin{array}{c}3.3666 e-5 \\
+1.4966 e-6 i\end{array}$ & $\begin{array}{c}3.3666 e-5 \\
-1.4966 e-6 i\end{array}$ & $\begin{array}{l}-1.7466 e-4 \\
-1.1910 e-5 i\end{array}$ & $\begin{array}{l}-1.7466 e-4 \\
+1.1910 e-5 i\end{array}$ & $\begin{array}{l}-1.0552 e-3 \\
+5.7213 e-5 i\end{array}$ \\
\hline & $7.3452 e-1$ & $7.3452 e-1$ & $9.0427 e-1$ & $9.0427 e-1$ & $4.6611 e-1$ \\
\hline & $\begin{array}{l}-6.4586 e-1 \\
-8.1957 e-4 i\end{array}$ & $\begin{array}{l}-6.4586 e-1 \\
+8.1957 e-4 i\end{array}$ & $\begin{array}{c}2.3200 e-1 \\
-3.7356 e-3 i\end{array}$ & $\begin{array}{c}2.3200 e-1 \\
+3.7356 e-3 i\end{array}$ & $\begin{array}{c}4.5189 e-1 \\
-6.5990 e-4 i\end{array}$ \\
\hline & $\begin{array}{c}1.8114 e-1 \\
+2.3984 e-3 i\end{array}$ & $\begin{array}{c}1.8114 e-1 \\
-2.3984 e-3 i\end{array}$ & $\begin{array}{l}-3.1975 e-1 \\
-3.6663 e-3 i\end{array}$ & $\begin{array}{l}-3.1975 e-1 \\
+3.6663 e-3 i\end{array}$ & $\begin{array}{c}4.2755 e-1 \\
-1.7674 e-3 i\end{array}$ \\
\hline & $\begin{array}{l}-1.5236 e-5 \\
+3.2631 e-4 i\end{array}$ & $\begin{array}{l}-1.5236 e-5 \\
-3.2631 e-4 i\end{array}$ & $\begin{array}{c}7.6868 e-5 \\
-1.0646 e-3 i\end{array}$ & $\begin{array}{c}7.6868 e-5 \\
+1.0646 e-3 i\end{array}$ & $\begin{array}{l}-4.0125 e-5 \\
-1.3052 e-3 i\end{array}$ \\
\hline & $\begin{array}{l}-1.5236 e-5 \\
+3.2631 e-4 i\end{array}$ & $\begin{array}{l}-1.5236 e-5 \\
-3.2631 e-4 i\end{array}$ & $\begin{array}{c}7.6868 e-5 \\
-1.0646 e-3 i \\
\end{array}$ & $\begin{array}{c}7.6868 e-5 \\
+1.0646 e-3 i \\
\end{array}$ & $\begin{array}{l}-4.0125 e-5 \\
-1.3052 e-3 i\end{array}$ \\
\hline \multirow{11}{*}{ First-order derivatives } & $\begin{array}{c}4.9349 e-3 \\
+4.1090 e-3 i\end{array}$ & $\begin{array}{c}1.8620 e-4 \\
+1.6593 e-6 i\end{array}$ & $\begin{array}{l}-7.1896 e-6 \\
-1.0523 e-6 i\end{array}$ & $\begin{array}{l}-6.7317 e-6 \\
-3.0627 e-7 i\end{array}$ & $\begin{array}{l}-3.0855 e-6 \\
+3.7924 e-5 i\end{array}$ \\
\hline & $\begin{array}{c}1.0543 e-2 \\
+4.9187 e-3 i\end{array}$ & $\begin{array}{c}3.1679 e-4 \\
-8.3544 e-5 i\end{array}$ & $\begin{array}{l}-7.4393 e-6 \\
-1.9432 e-6 i\end{array}$ & $\begin{array}{l}-4.9700 e-6 \\
+5.0936 e-7 i\end{array}$ & $\begin{array}{l}-3.9019 e-6 \\
+3.1021 e-5 i\end{array}$ \\
\hline & $\begin{array}{c}6.9776 e-3 \\
-1.5213 e-3 i\end{array}$ & $\begin{array}{c}1.2503 e-4 \\
-1.5632 e-4 i\end{array}$ & $\begin{array}{l}-9.1416 e-6 \\
-3.4699 e-6 i\end{array}$ & $\begin{array}{l}-6.7761 e-7 \\
+3.0202 e-6 i\end{array}$ & $\begin{array}{l}-5.3973 e-6 \\
+2.1570 e-5 i\end{array}$ \\
\hline & $\begin{array}{c}1.2865 e-8 \\
-5.3235 e-8 i\end{array}$ & $\begin{array}{l}-6.7567 e-6 \\
-1.3890 e-7 i\end{array}$ & $\begin{array}{c}4.3822 e-4 \\
-7.3751 e-3 i\end{array}$ & $\begin{array}{c}4.3945 e-4 \\
-7.3752 e-3 i\end{array}$ & $\begin{array}{l}-4.1661 e-2 \\
-2.4195 e-3 i\end{array}$ \\
\hline & $\begin{array}{l}-1.2865 e-8 \\
+5.3235 e-8 i\end{array}$ & $\begin{array}{c}6.7567 e-6 \\
+1.3890 e-7 i\end{array}$ & $\begin{array}{c}4.3822 e-4 \\
-7.3751 e-3 i\end{array}$ & $\begin{array}{c}4.3945 e-4 \\
-7.3752 e-3 i\end{array}$ & $\begin{array}{l}-4.1661 e-2 \\
-2.4195 e-3 i\end{array}$ \\
\hline & $\begin{array}{l}-4.0427 e-2 \\
-1.5051 e-2 i\end{array}$ & $\begin{array}{l}-1.1609 e-3 \\
+4.0699 e-4 i\end{array}$ & $\begin{array}{c}4.9009 e-6 \\
+2.4447 e-5 i\end{array}$ & $\begin{array}{l}-2.3870 e-6 \\
+1.5967 e-5 i\end{array}$ & $\begin{array}{c}1.1140 e-4 \\
+7.8994 e-6 i\end{array}$ \\
\hline & $\begin{array}{l}-2.3209 e-2 \\
+3.7847 e-2 i\end{array}$ & $\begin{array}{c}1.9018 e-4 \\
+1.2372 e-3 i\end{array}$ & $\begin{array}{c}1.7448 e-5 \\
+2.6237 e-5 i\end{array}$ & $\begin{array}{l}-1.1561 e-5 \\
+4.3792 e-6 i\end{array}$ & $\begin{array}{l}9.9583 e-5 \\
+1.0880 e-5 i\end{array}$ \\
\hline & $\begin{array}{c}1.2020 e-2 \\
+5.5792 e-3 i\end{array}$ & $\begin{array}{c}3.5070 e-4 \\
-9.4669 e-5 i\end{array}$ & $\begin{array}{l}-1.0988 e-5 \\
+2.4234 e-5 i\end{array}$ & $\begin{array}{c}1.4771 e-5 \\
+4.6761 e-6 i\end{array}$ & $\begin{array}{c}5.8350 e-5 \\
-1.5286 e-5 i\end{array}$ \\
\hline & $\begin{array}{c}1.4840 e-9 \\
+4.4428 e-8 i\end{array}$ & $\begin{array}{c}4.0257 e-7 \\
-1.6972 e-7 i\end{array}$ & $\begin{array}{l}-2.0897 e-2 \\
-6.1056 e-5 i\end{array}$ & $\begin{array}{l}-2.0897 e-2 \\
-5.7389 e-5 i\end{array}$ & $\begin{array}{l}-1.6748 e-4 \\
+1.1803 e-1 i\end{array}$ \\
\hline & $\begin{array}{l}-1.4840 e-9 \\
-4.4428 e-8 i\end{array}$ & $\begin{array}{l}-4.0257 e-7 \\
+1.6972 e-7 i \\
\end{array}$ & $\begin{array}{l}-2.0897 e-2 \\
-6.1056 e-5 i\end{array}$ & $\begin{array}{l}-2.0897 e-2 \\
-5.7389 e-5 i\end{array}$ & $\begin{array}{l}-1.6748 e-4 \\
+1.1803 e-1 i\end{array}$ \\
\hline & 6 & 7 & 8 & 9 & 10 \\
\hline \multirow{6}{*}{$\begin{array}{l}\text { Generalized } \\
\text { eigenvectors }\end{array}$} & $\begin{array}{l}-8.8391 e-3 \\
+3.7710 e-1 i\end{array}$ & $\begin{array}{c}2.1782 e-4 \\
-1.6872 e-5 i\end{array}$ & $\begin{array}{c}2.1782 e-4 \\
+1.6872 e-5 i\end{array}$ & $\begin{array}{l}-2.1190 e-17 \\
-2.9349 e-18 i\end{array}$ & $\begin{array}{l}-2.1190 e-17 \\
+2.9349 e-18 i\end{array}$ \\
\hline & $\begin{array}{l}-9.1033 e-3 \\
+3.6559 e-1 i\end{array}$ & $\begin{array}{c}1.8288 e-4 \\
-1.8087 e-5 i\end{array}$ & $\begin{array}{c}1.8288 e-4 \\
+1.8087 e-5 i\end{array}$ & $\begin{array}{l}-1.6163 e-17 \\
-6.5559 e-19 i\end{array}$ & $\begin{array}{l}-1.6163 e-17 \\
+6.5559 e-19 i\end{array}$ \\
\hline & $\begin{array}{l}-9.5377 e-3 \\
+3.4587 e-1 i\end{array}$ & $\begin{array}{c}1.2866 e-4 \\
-1.9205 e-5 i\end{array}$ & $\begin{array}{c}1.2866 e-4 \\
+1.9205 e-5 i\end{array}$ & $\begin{array}{l}-1.3628 e-17 \\
-1.3204 e-17 i\end{array}$ & $\begin{array}{l}-1.3628 e-17 \\
+1.3204 e-17 i\end{array}$ \\
\hline & $\begin{array}{l}-1.0552 e-3 \\
-5.7213 e-5 i\end{array}$ & $\begin{array}{l}-1.3333 e-2 \\
-2.3533 e-1 i\end{array}$ & $\begin{array}{l}-1.3333 e-2 \\
+2.3533 e-1 i\end{array}$ & $\begin{array}{c}1.2667 e-2 \\
+2.3536 e-1 i\end{array}$ & $\begin{array}{c}1.2667 e-2 \\
-2.3536 e-1 i\end{array}$ \\
\hline & $\begin{array}{l}-1.0552 e-3 \\
-5.7213 e-5 i\end{array}$ & $\begin{array}{l}-1.3333 e-2 \\
-2.3533 e-1 i\end{array}$ & $\begin{array}{l}-1.3333 e-2 \\
+2.3533 e-1 i\end{array}$ & $\begin{array}{l}-1.2667 e-2 \\
-2.3536 e-1 i\end{array}$ & $\begin{array}{l}-1.2667 e-2 \\
+2.3536 e-1 i\end{array}$ \\
\hline & $4.6611 e-1$ & $\begin{array}{c}1.2794 e-5 \\
+6.1779 e-4 i\end{array}$ & $\begin{array}{c}1.2794 e-5 \\
-6.1779 e-4 i\end{array}$ & $\begin{array}{c}1.9126 e-17 \\
-2.8812 e-17 i\end{array}$ & $\begin{array}{c}1.9126 e-17 \\
+2.8812 e-17 i\end{array}$ \\
\hline
\end{tabular}


TABle 1: Continued.

\begin{tabular}{|c|c|c|c|c|c|}
\hline & \multicolumn{5}{|c|}{ Mode } \\
\hline & 6 & 7 & 8 & 9 & 10 \\
\hline & $\begin{array}{l}4.5189 e-1 \\
+6.5990 e-4 i\end{array}$ & $\begin{array}{l}2.1815 e-5 \\
+5.1933 e-4 i\end{array}$ & $\begin{array}{l}2.1815 e-5 \\
-5.1933 e-4 i\end{array}$ & $\begin{array}{c}2.7275 e-17 \\
+1.0443 e-16 i\end{array}$ & $\begin{array}{c}2.7275 e-17 \\
-1.0443 e-16 i\end{array}$ \\
\hline & $\begin{array}{c}4.2755 e-1 \\
+1.7674 e-3 i\end{array}$ & $\begin{array}{c}3.3649 e-5 \\
+3.6639 e-4 i\end{array}$ & $\begin{array}{c}3.3649 e-5 \\
-3.6639 e-4 i\end{array}$ & $\begin{array}{l}-5.0624 e-17 \\
+6.1754 e-17 i\end{array}$ & $\begin{array}{l}-5.0624 e-17 \\
-6.1754 e-17 i\end{array}$ \\
\hline & $\begin{array}{l}-4.0125 e-5 \\
+1.3052 e-3 i\end{array}$ & $\begin{array}{c}6.6667 e-1 \\
-6.1319 e-15 i\end{array}$ & $\begin{array}{c}6.6667 e-1 \\
+6.1319 e-15 i\end{array}$ & $\begin{array}{c}-6.6667 e-1 \\
+1.9880 e-14 i\end{array}$ & $\begin{array}{c}-6.6667 e-1 \\
-1.9880 e-14 i\end{array}$ \\
\hline & $\begin{array}{l}-4.0125 e-5 \\
+1.3052 e-3 i\end{array}$ & $6.6667 e-1$ & $6.6667 e-1$ & $6.6667 e-1$ & $6.6667 e-1$ \\
\hline \multirow{10}{*}{ First-order derivatives } & $\begin{array}{c}1.7349 e-4 \\
-1.6163 e-4 i\end{array}$ & $\begin{array}{c}3.9518 e-4 \\
-3.7717 e-4 i\end{array}$ & $\begin{array}{l}-6.6110 e-6 \\
-6.2260 e-7 i\end{array}$ & $\begin{array}{l}-1.3023 e-4 \\
+4.9260 e-5 i\end{array}$ & $\begin{array}{l}-3.0598 e-6 \\
+3.8496 e-5 i\end{array}$ \\
\hline & $\begin{array}{c}2.0752 e-4 \\
-3.4405 e-4 i\end{array}$ & $\begin{array}{c}4.7933 e-4 \\
-8.0841 e-4 i\end{array}$ & $\begin{array}{l}-4.6504 e-6 \\
-1.2688 e-6 i\end{array}$ & $\begin{array}{l}-3.0853 e-4 \\
+1.7329 e-4 i\end{array}$ & $\begin{array}{l}-3.4365 e-6 \\
+3.2083 e-5 i\end{array}$ \\
\hline & $\begin{array}{l}-1.9104 e-5 \\
-2.4330 e-4 i\end{array}$ & $\begin{array}{l}-5.3099 e-5 \\
-5.6976 e-4 i\end{array}$ & $\begin{array}{l}-1.2556 e-7 \\
-4.0572 e-6 i\end{array}$ & $\begin{array}{l}-6.5242 e-5 \\
+2.1995 e-4 i\end{array}$ & $\begin{array}{l}-4.4476 e-6 \\
+2.2222 e-5 i\end{array}$ \\
\hline & $\begin{array}{l}-1.7809 e-7 \\
-6.5745 e-6 i\end{array}$ & $\begin{array}{l}-4.1770 e+7 \\
-1.4953 e+7 i\end{array}$ & $\begin{array}{c}4.3894 e-4 \\
-7.3760 e-3 i\end{array}$ & $\begin{array}{c}1.1347 e-7 \\
+1.1973 e-7 i\end{array}$ & $\begin{array}{l}-4.1661 e-2 \\
-2.4196 e-3 i\end{array}$ \\
\hline & $\begin{array}{c}1.7809 e-7 \\
+6.5745 e-6 i\end{array}$ & $\begin{array}{c}4.1770 e-7 \\
+1.4953 e-7 i\end{array}$ & $\begin{array}{c}4.3894 e-4 \\
-7.3760 e-3 i\end{array}$ & $\begin{array}{l}-1.1347 e-7 \\
-1.1973 e-7 i\end{array}$ & $\begin{array}{l}-4.1661 e-2 \\
-2.4196 e-3 i\end{array}$ \\
\hline & $\begin{array}{l}-6.8458 e-4 \\
+1.3706 e-3 i\end{array}$ & $\begin{array}{l}-1.5683 e-3 \\
+3.1981 e-3 i\end{array}$ & $\begin{array}{l}-3.6769 e-6 \\
+2.1007 e-5 i\end{array}$ & $\begin{array}{c}8.6146 e-4 \\
-6.5660 e-4 i\end{array}$ & $\begin{array}{c}1.0981 e-4 \\
+3.3384 e-6 i\end{array}$ \\
\hline & $\begin{array}{c}1.2345 e-3 \\
+9.1495 e-4 i\end{array}$ & $\begin{array}{c}2.8941 e-3 \\
+2.1296 e-3 i\end{array}$ & $\begin{array}{l}-1.3319 e-5 \\
+2.6782 e-5 i\end{array}$ & $\begin{array}{l}-3.5926 e-4 \\
-1.2558 e-3 i\end{array}$ & $\begin{array}{c}9.4929 e-5 \\
+6.8591 e-6 i\end{array}$ \\
\hline & $\begin{array}{c}2.1805 e-4 \\
-3.7015 e-4 i\end{array}$ & $\begin{array}{c}5.1275 e-4 \\
-8.8146 e-4 i\end{array}$ & $\begin{array}{l}-5.2960 e-6 \\
-1.9547 e-6 i\end{array}$ & $\begin{array}{l}-4.6276 e-4 \\
+2.3522 e-4 i\end{array}$ & $\begin{array}{c}6.2620 e-5 \\
-7.5296 e-6 i\end{array}$ \\
\hline & $\begin{array}{c}2.1276 e-7 \\
-4.9301 e-7 i\end{array}$ & $\begin{array}{c}4.8581 e-7 \\
-1.1570 e-8 i\end{array}$ & $\begin{array}{l}-2.0895 e-2 \\
-5.8814 e-5 i\end{array}$ & $\begin{array}{l}-3.5540 e-7 \\
+3.0228 e-7 i\end{array}$ & $\begin{array}{l}-1.6732 e-4 \\
+1.1803 e-1 i\end{array}$ \\
\hline & $\begin{array}{l}-2.1276 e-7 \\
+4.9301 e-7 i\end{array}$ & $\begin{array}{l}-4.8581 e-7 \\
+1.1570 e-8 i\end{array}$ & $\begin{array}{l}-2.0895 e-2 \\
-5.8814 e-5 i\end{array}$ & $\begin{array}{c}3.5540 e-7 \\
-3.0228 e-7 i\end{array}$ & $\begin{array}{l}-1.6732 e-4 \\
+1.1803 e-1 i\end{array}$ \\
\hline
\end{tabular}

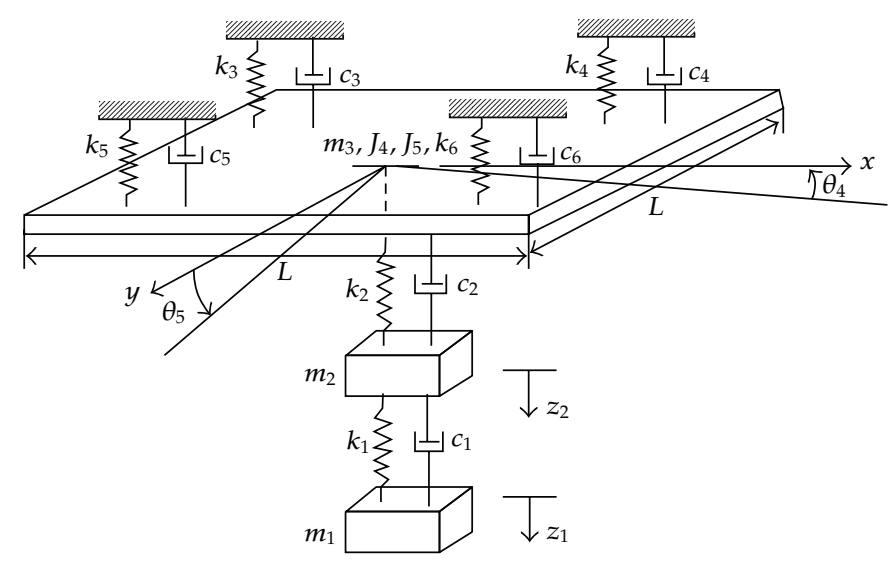

FIGURE 1: 5-DOF spring-mass system. 


$$
\begin{aligned}
& \text { Suppose } \\
& \begin{array}{c}
m_{1}=300 \mathrm{~kg}, \quad m_{2}=750 \mathrm{~kg}, \quad m_{3}=1500 \mathrm{~kg}, \\
k_{1}=15000 \mathrm{~N} / \mathrm{m}, \quad k_{2}=30000 \mathrm{~N} / \mathrm{m}, \\
k_{3}=1000 \mathrm{~N} / \mathrm{m}, \quad k_{4}=1000 \mathrm{~N} / \mathrm{m}, \\
k_{5}=1000 \mathrm{~N} / \mathrm{m}, \quad k_{6}=1000 \mathrm{~N} / \mathrm{m}, \\
c_{1}=6 \mathrm{Ns} / \mathrm{m}, \quad c_{2}=9 \mathrm{Ns} / \mathrm{m}, \\
c_{3}=40 \mathrm{Ns} / \mathrm{m}, \quad c_{4}=40 \mathrm{Ns} / \mathrm{m}, \\
c_{5}=40 \mathrm{Ns} / \mathrm{m}, \quad c_{6}=40 \mathrm{Ns} / \mathrm{m} .
\end{array}
\end{aligned}
$$

Thus,

$$
\begin{aligned}
& \mathbf{M}=\left(\begin{array}{ccccc}
300 & 0 & 0 & 0 & 0 \\
0 & 750 & 0 & 0 & 0 \\
0 & 0 & 1500 & 0 & 0 \\
0 & 0 & 0 & 125 & 0 \\
0 & 0 & 0 & 0 & 125
\end{array}\right) \\
& \mathbf{K}=\left(\begin{array}{ccccc}
15000 & -15000 & 0 & 0 & 0 \\
-15000 & 45000 & -30000 & 0 & 0 \\
0 & 0 & 34000 & 0 & 0 \\
0 & 0 & 0 & 1000 & 0 \\
0 & 0 & 0 & 0 & 1000
\end{array}\right) \text {, } \\
& \mathbf{C}=\left(\begin{array}{ccccc}
6 & -6 & 0 & 0 & 0 \\
-6 & 15 & -9 & 0 & 0 \\
0 & -9 & 169 & 0 & 0 \\
0 & 0 & 0 & 40 & 0 \\
0 & 0 & 0 & 0 & 40
\end{array}\right)
\end{aligned}
$$

The system has two 2-repeated eigenvalues: $-0.1600+$ $2.8239 i$, and $-0.1600-2.8239 i$ and distinct eigenvalues $-0.0218 \pm 9.6935 i,-0.0248 \pm 6.0969 i,-0.0297 \pm 1.2353 i$. Here, the damper $c_{6}$ is taken as the design parameter. Evaluation of eigenvectors and the first-order derivatives with respect to $c_{6}$ will be illustrated for $\Delta c_{6} / c_{6}=0.1$ in Table 1 .

\section{Conclusion}

The extension of CA method is outlined to enable the calculation of eigenvectors sensitivity analysis for general linear damped vibration systems with repeated eigenvalues. CA method developed recently is suitable for efficientaccurate evaluation of the structural response at various modified designs. The difficulty of applying the CA approach to calculate the first-order derivatives is the singularity of characteristic matrix. A relaxation factor embedded in the CA method is used to keep the reversibility. And this makes it possible and effective to deal with the problems of sensitivity analysis for systems with multiple eigenvalues. Unlike common procedures of structural response, the approach proposed is not based on calculation of derivatives. Rather, approximations of modified eigenvectors are used to evaluate the modified first-order derivatives. Similar computational procedures are employed for evaluating eigenvectors and first-order derivatives of eigenvectors. The presented approach here is simple and general in nature. A numerical example of 5-DOF spring-mass system demonstrates the accuracy and efficiency of the proposed method.

\section{Acknowledgments}

The Grant of the projects from the National Natural Science Foundation of China (no. 51205159) and Doctoral Program of Higher Education (no. 20090061110022) and Major Program of Science and Technology Development Plan of Jilin Province of China (no. 20126001) and Graduate Innovation Fund of Jilin University (no. 20121097) is gratefully acknowledged as a form of the financial support.

\section{References}

[1] J. E. Mottershead and M. I. Friswell, "Model updating in structural dynamics: a survey," Journal of Sound and Vibration, vol. 167, no. 2, pp. 347-375, 1993.

[2] A. Messina, E. J. Williams, and T. Contursi, "Structural damage detection by a sensitivity and statistical-based method," Journal of Sound and Vibration, vol. 216, no. 5, pp. 791-808, 1998.

[3] J. H. Wilkinson, The Algebraic Eigenvalue Problem, Oxford University Press, New York, NY, USA, 1965.

[4] R. L. Fox and M. P. Kapoor, "Rates of change of eigenvalues and eigenvectors," The American Institute of Aeronautics and Astronautics, vol. 6, no. 12, pp. 2426-2429, 1968.

[5] L. C. Rogers, "Derivatives of eigenvalues and eigenvectors," The American Institute of Aeronautics and Astronautics, vol. 8, pp. 943-944, 1970

[6] R. B. Nelson, "Simplified calculation of eigenvector derivatives," The American Institute of Aeronautics and Astronautics, vol. 14, no. 9, pp. 1201-1205, 1976.

[7] I. U. Ojalvo, "Efficient computation of modal sensitivities for systems with repeated frequencies," The American Institute of Aeronautics and Astronautics, vol. 26, no. 3, pp. 361-366, 1988.

[8] W. C. Mills-Curran, "Calculation of eigenvector derivatives for structures with repeated eigenvalues," The American Institute of Aeronautics and Astronautics, vol. 26, no. 7, pp. 867-871, 1988.

[9] R. L. Dailey, "Eigenvector derivatives with repeated eigenvalues," The American Institute of Aeronautics and Astronautics, vol. 27, no. 4, pp. 486-491, 1989.

[10] W. C. Mills-Curran, "Comment on "eigenvector derivatives with repeated eigenvalues"', The American Institute of Aeronautics and Astronautics, vol. 28, no. 10, p. 1846, 1990.

[11] I. W. Lee, G. H. Jung, and J. W. Lee, "Numerical method for sensitivity analysis of eigensystems with non-repeated and repeated eigenvalues," Journal of Sound and Vibration, vol. 195, no. 1, pp. 17-32, 1996.

[12] S. H. Chen, Matrix Perturbation Theory in Structural Dynamics, Science Press, Beijing, China, 2007.

[13] U. Kirsch, "Combined approximations-a general reanalysis approach for structural optimization," Structural and Multidisciplinary Optimization, vol. 20, no. 2, pp. 97-106, 2000.

[14] U. Kirsch, M. Bogomolni, and I. Sheinman, "Efficient procedures for repeated calculations of structural sensitivities," Engineering Optimization, vol. 39, no. 3, pp. 307-325, 2007. 


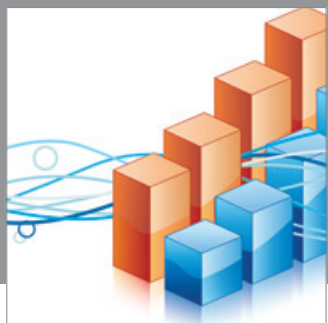

Advances in

Operations Research

mansans

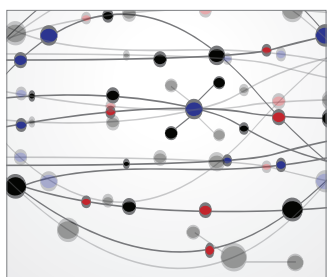

The Scientific World Journal
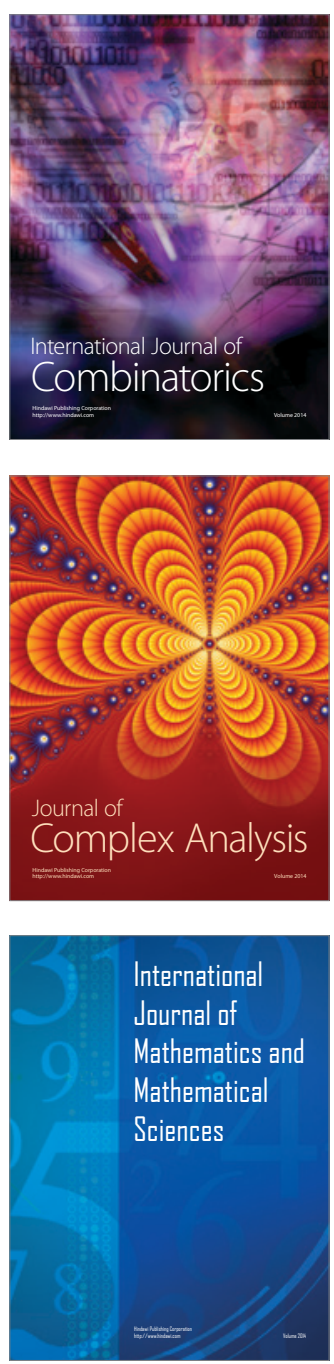
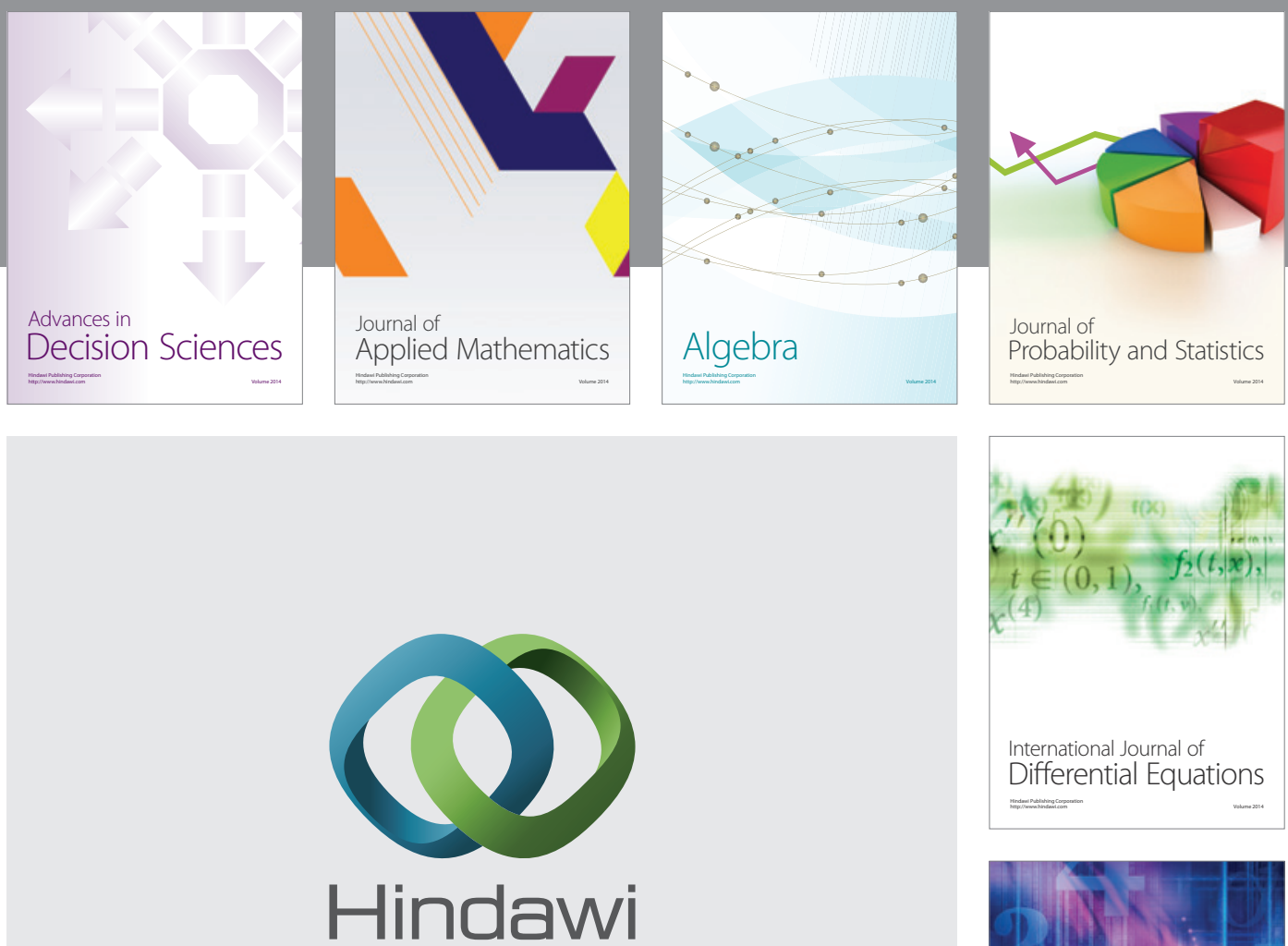

Submit your manuscripts at http://www.hindawi.com
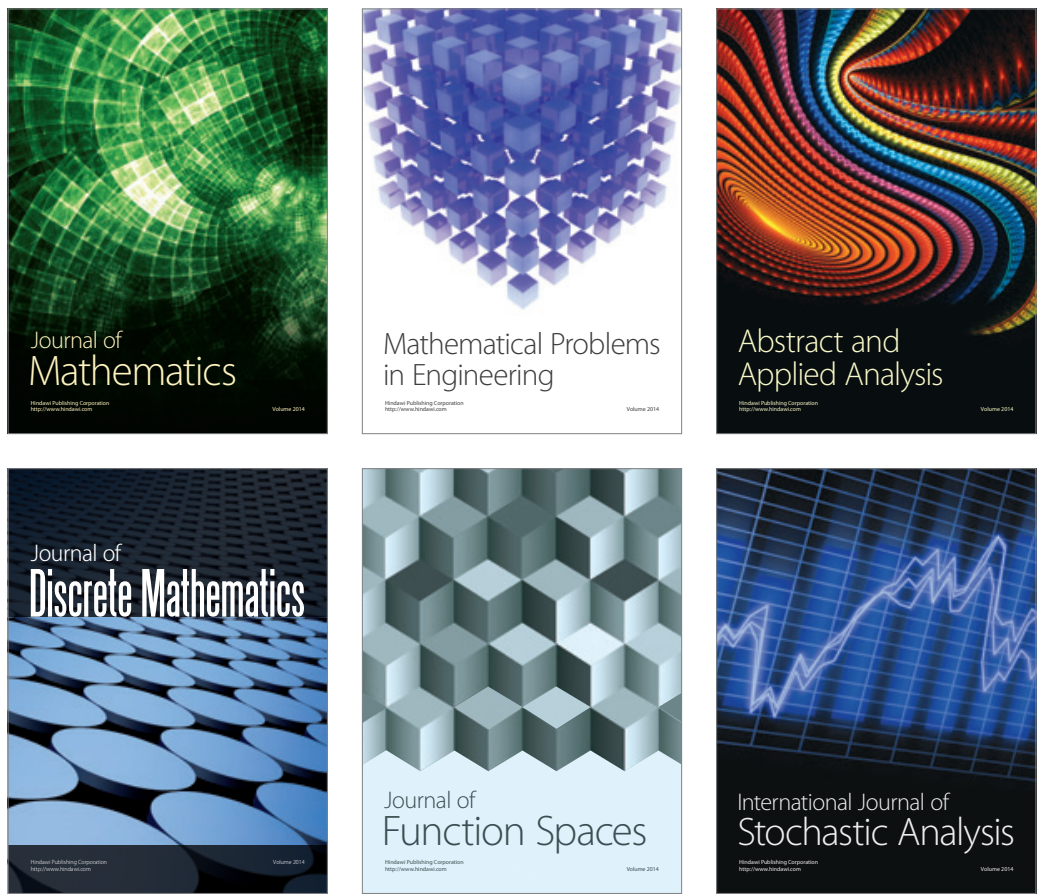

Journal of

Function Spaces

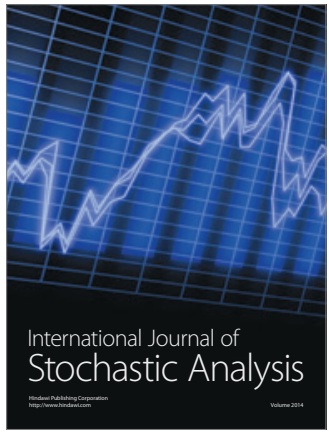

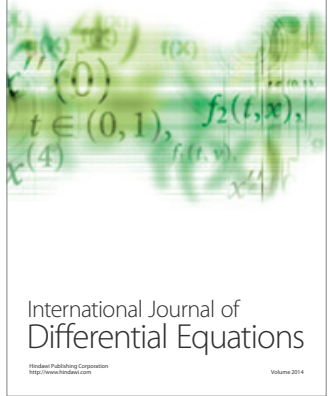
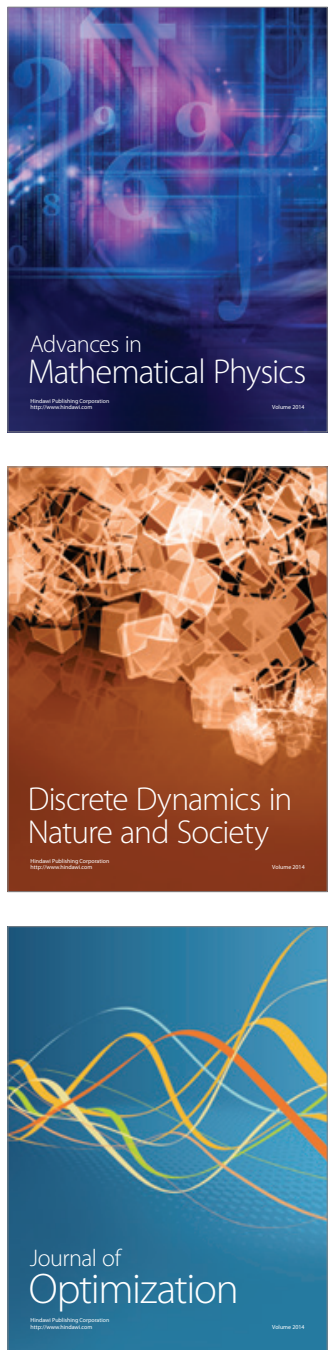